www.nature.com/jhg

\title{
A genome-wide association study identifies SNP in $D C C$ is associated with gallbladder cancer in the Japanese population
}

\author{
Pei-Chieng Cha ${ }^{1}$, Hitoshi Zembutsu ${ }^{1}$, Atsushi Takahashi ${ }^{2}$, Michiaki Kubo ${ }^{3}$, Naoyuki Kamatani ${ }^{4}$ and \\ Yusuke Nakamura ${ }^{1}$
}

Gallbladder cancer (GC) is a relatively uncommon cancer with higher incidence in certain areas including Japan. Because of the difficulty in diagnosis, prognosis of GC is very poor. To identify genetic determinants of GC, we conducted a genome-wide association study (GWAS) in $41 \mathrm{GC}$ patients and 866 controls. Association between each single-nucleotide polymorphism (SNP) with GC susceptibility was evaluated by multivariate logistic regression analysis conditioned on age and gender of subjects. SNPs that showed suggestive association $\left(P<1 \times 10^{-4}\right)$ with GC were further examined in 30 cases and 898 controls. SNP rs7504990 in the DCC (deleted in colorectal cancer, 18q21.3) that encodes a netrin 1 receptor achieved a combined $P$-value of $7.46 \times 10^{-8}(\mathrm{OR}=6.95 ; 95 \% \mathrm{Cl}=3.43-14.08)$. Subsequent imputation analysis identified multiple SNPs with similarly strong associations in an adjacent genomic region, where loss of heterozygosity was reported in GC and other cancers. Reduced expression of $D C C$ was indicated to be associated with the poorly differentiated histological type, increased proliferation and metastasis through loss of adhesiveness. However, due to the limited sample size investigated here, further replication study and functional analysis would be necessary to further confirm the result of the association.

Journal of Human Genetics (2012) 57, 235-237; doi:10.1038/jhg.2012.9; published online 9 February 2012

Keywords: DCC; GWAS; gallbladder cancer; SNP

\section{INTRODUCTION}

Gallbladder cancer (GC) is the most common malignancy of the biliary tract and the fifth most common cancer of the gastrointestinal tract. ${ }^{1}$ With an overall 5 -year survival rate of $<5 \%$, GC is a highly lethal malignancy with very poor prognosis. ${ }^{2}$ Most of GCs were diagnosed at a very late stage because of the lack of symptoms and non-specific symptoms of early-stage tumors. ${ }^{1,3}$ Although the incidence of GC is relatively rare compared with other cancers, higher incidences of GC have been reported in certain geographical regions including India, Pakistan, Ecuador, Korea and Japan. ${ }^{4}$ In addition, the prevalence of GC is known to be three times higher in women than in men. ${ }^{5}$ Although several clinical risk factors of GC such as gallstones, cholecystitis, porcelain gallbladder, gallbladder polyps, anomalous panreatobiliary duct junction and obesity have been indicated, ${ }^{1,6}$ etiology of GC is largely unknown. Only few somatic genetic changes including mutations in K-ras, TP53 and p16 $6^{\text {Ink4 } / C D K N 2 ~ a s ~ w e l l ~ a s ~ l o s s ~ o f ~ h e t e r o z y g o s i t y ~ a t ~ s e v e r a l ~ c h r o m o-~}$ somal regions harboring known or putative tumor-suppressor genes have been reported in GCs. ${ }^{5,7}$ Hence, identification of novel genetic factors associated with susceptibility to GC should provide new insights into pathogenesis and novel therapeutic interventions of GC. Here, we report a genome-wide association study (GWAS) that aims to identify genetic factors associated with GC susceptibility.

\section{MATERIALS AND METHODS}

Subjects

In the GWAS, 41 patients who were diagnosed to have GC and 866 control subjects were examined. All cases were registered into Biobank Japan supported by the Ministry of Education, Culture, Sports, Science and Technology, Japan (http://www.biobankjp.org/). Controls consisted of healthy volunteers from Osaka-Midosuji Rotary Club. In the replication study, 30 GC patients and 898 controls were investigated. All cases and controls in the replication study were obtained from Biobank Japan. All control subjects do not have medical history of GC or gallstones, cholecystitis and other known confounding diseases for GC. Among the 71 cases, 45 were adenocarcimas and one was a squamous cell carcinoma, and the histological information for the remaining ones was not obtained. All subjects had given written informed consent to participate in the study in accordance with the process approved by Ethical Committee at the Institute of Medical Science of the University of Tokyo and Center for Genomic Medicine of RIKEN. Demographical information of subjects was summarized in Supplementary Table 1.

${ }^{1}$ Institute of Medical Science, Laboratory of Molecular Medicine, Human Genome Center, The University of Tokyo, Tokyo, Japan; ${ }^{2}$ Laboratory for Statistical Analysis, RIKEN Center for Genomic Medicine, Yokohama, Japan; ${ }^{3}$ Laboratory for Genotyping Development, RIKEN Center for Genomic Medicine, Yokohama, Japan and ${ }^{4}$ Research Groups for Pharmacogenomics, RIKEN Center for Genomic Medicine, Yokohama, Japan

Correspondence: Professor Y Nakamura, Laboratory of Molecular Medicine, Human Genome Center, The University of Tokyo, 4-6-1 Shirokanedai, Minato-ku, Tokyo 108-8639, Japan. 


\section{Genotyping and quality control}

All cases and controls in the GWAS were genotyped by using the Illumina HumanHap550 Genotyping BeadChip (San Diego, CA, USA). QC of genotyping data was performed whereby subjects with a call rate of $<98 \%$, singlenucleotide polymorphisms (SNPs) with a call rate of $<99 \%$ or minor allele frequency of $<0.01$, as well as SNPs with a Hardy-Weinberg equilibrium test's $P$-value of $<1 \times 10^{-6}$ were excluded from the subsequent statistical analysis. In the replication study, cases and controls were genotyped by using the Illumina HumanHap610 Genotyping BeadChip (San Diego).

\section{Statistical analysis}

Association of each SNP with susceptibility to GC was evaluated by logistic regression analysis conditioned on age and gender of subjects. The significance of association was evaluated based on the minimum $P$-value among the additive, dominant and recessive model of inheritance. SNPs that showed a minimum $P$-value of $<1 \times 10^{-4}$ in the GWAS were considered as showing suggestive association with GC and were examined in additional subjects. QC and statistical analysis were performed by using the PLINK statistical software (v1.06) (http://pngu.mgh.harvard.edu/ purcell/plink/). ${ }^{8}$

\section{Imputation}

Imputation analysis was performed based on genotype of Japanese (JPT) individuals in the Phase II HapMap database (release 24) by using software MACH v1.0 (http://www.sph.umich.edu/csg/yli/mach/index.html). ${ }^{9} \quad$ SNPs located in the genomic region within $1500 \mathrm{~kb}$ upstream and downstream of the marker SNP, which showed the strongest association with GC, was imputed by implementing 50 Markov Chain iterations. As a QC measure, imputed SNPs with an imputation quality score of $<0.3$ were excluded from the subsequent association analysis. Pair-wise linkage disequilibrium $\left(r^{2}\right)$ between each SNP with the marker SNP was estimated by using the PLINK statistical software (v1.06); whereas regional association plot was generated by the $\mathrm{R}$ program v2.10.0 (http://www.r-project.org/). Possible functional consequences of SNPs were predicted in silico by using the SNPinfo web server (http://manticore. niehs.nih.gov/cgi-bin/snpinfo/snpfunc.cgi). eQTL analysis was performed based on data available from the Sanger Institute GENEVAR project ${ }^{10}$ for lymphoblastoid cell lines from the four HapMap populations.

\section{RESULTS}

In this GWAS, 425706 SNPs with a total genotyping rate of $>99 \%$ in 41 GC patients and 866 controls were analyzed after QC of the genotyping data. A genomic inflation factor $(\lambda)$ in the quantilequantile plot (Supplementary Figure 1a) was calculated to be 0.9903 , implying low possibility of false-positive associations resulting from the population stratification or cryptic relatedness. The Manhattan plot (Supplementary Figure 1b) indicated that none of the genotyped SNPs achieved genome-wide significant association $\left(P<1 \times 10^{-7}\right)$ with GC. However, 130 SNPs showed suggestive association with a minimum $P$-value of $<1 \times 10^{-4}$. These SNPs were examined in the additional 30 GC patients and 898 controls, and the genotyping results are shown in Supplementary Table 2. Among these SNPs, SNP rs7504990 that is located in the DCC (deleted in colorectal cancer) achieved a minimum $P$-value of $9.67 \times 10^{-5}$ under the recessive model of inheritance in the replication study. As illustrated in Table 1, this SNP achieved a $P$-value of $7.46 \times 10^{-8}(\mathrm{OR}=6.95$; 95\% CI $=3.43-$ 14.08) in the combined study. Genotype AA the risk genotype, was found to be enriched in cases than in the controls, with a genotype frequency of 0.19 versus 0.04 . Interestingly, the SNP that showed the second lowest $P$-value, SNP rs4078288, is also located in the DCC (Table 1). To further characterize the association of the $D C C$ region with the GC risk, genotypes of SNPs located in the genomic region within $1500 \mathrm{~kb}$ upstream and downstream of the marker SNP rs7504990 were imputed. Association analysis for the imputed and genotyped SNPs with GC susceptibility was then conducted. Figure 1 illustrates the regional plot for association results of the investigated

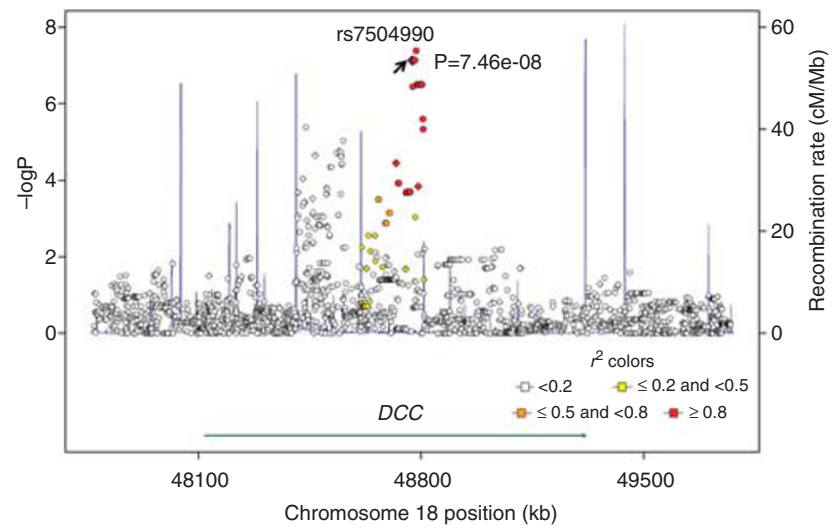

Figure 1 Regional plot for associations of SNPs located within $1500 \mathrm{~kb}$ upstream and downstream of the marker SNP, rs7504990, with gallbladder cancer (GC). The $-\log _{10}(P)$ values of SNPs were plotted against relative chromosomal locations. Diamond and circle signs represent genotyped and imputed SNPs, respectively. All SNPs are color-coded as red (0.8-1.0), orange $(0.5-0.8)$, yellow $(0.2-0.5)$ and white $(<0.2)$, according to their pairwise $r^{2}$ to the marker SNP. The marker SNP is indicated by an arrow and the combined $P$-value of the marker SNP is represented by a blue diamond sign. SNP positions followed NCBI build 36 coordinates. Estimated recombination rates $(\mathrm{cM} / \mathrm{Mb})$ were plotted in dark blue line.

SNPs. We observed that multiple SNPs in the genomic region adjacent to the marker SNP also showed associations, as strong as or stronger than the marker SNP, with GC susceptibility. SNPs that showed $P$-values of $<1 \times 10^{-6}$ with GC susceptibility were listed in Supplementary Table 3. All these SNPs are located in intronic regions of the DCC. Online prediction by using the SNPinfo web server (http:// manticore.niehs.nih.gov/cgi-bin/snpinfo/snpfunc.cgi) revealed that none of these SNPs was likely to alter transcription factor binding site. In addition, none of them was linked to exonic SNPs of the DCC with an $r^{2}$ value of 0.8 or over. We examined eQTL data for all SNPs in Supplementary Table 3 that showed strong association with GC $\left(P<1 \times 10^{-6}\right)$ and found that none of them potentially altered expression of DCC in the four investigated HapMap populations.

\section{DISCUSSION}

Through the current GWAS and the subsequent replication study, we report here that SNP rs7504990 in the DCC showed genome-wide significant association with GC susceptibility $\left(P_{\text {Combined }}=7.46 \times 10^{-8}\right.$; $\mathrm{OR}=6.95 ; 95 \% \mathrm{CI}=3.43-14.08)$ in the Japanese population. Subsequent imputation and association analysis revealed additional SNPs located adjacent to the marker SNP rs7504990 in the DCC to be associated with GC susceptibility. Regional association plot indicated that SNPs showing strong association with GC are located in one linkage disequilibrium block that was not disrupted by recombination event. Results from subsequent in silico analysis and eQTL analysis are concordant, whereby none of these SNPs potentially alter transcription factor binding site nor possibly alter the expression of DCC.

$D C C$ encodes a netrin 1 receptor, which is a transmembrane protein that is a member of the immunoglobulin superfamily of cell adhesion molecules. ${ }^{11}$ Loss of heterozygosity and microsatellite instability in the chromosome 18q21 region that contains the DCC have been observed in multiple cancer types, particularly cancers in the digestive organ including colon, ${ }^{12}$ stomach, ${ }^{13}$ esophagus, ${ }^{14}$ pancreas, ${ }^{15}$ and gallbladder. ${ }^{16}$ In addition, reduced or loss of the DCC expression has been associated with the poorly differentiated histological type, increased proliferation and metastasis through the loss of adhesiveness. ${ }^{17}$ 
Table 1 SNPs in DCC associated with GC

\begin{tabular}{|c|c|c|c|c|c|c|c|c|c|c|c|c|c|c|c|c|}
\hline \multirow{2}{*}{$\begin{array}{l}\text { Chromosome } \\
\text { no. }\end{array}$} & \multirow{2}{*}{$\begin{array}{c}\text { Chromosome } \\
\text { location }\end{array}$} & \multirow[b]{2}{*}{ SNP } & \multirow[b]{2}{*}{$A 1$} & \multirow[b]{2}{*}{ Stages } & \multicolumn{4}{|c|}{ Cases } & \multicolumn{4}{|c|}{ Controls } & \multirow[b]{2}{*}{ Minimum $P$} & \multirow[b]{2}{*}{$O R$} & \multirow[b]{2}{*}{$L 95$} & \multirow[b]{2}{*}{ U95 } \\
\hline & & & & & $p(11)$ & $p(12)$ & $p(22)$ & MAF & $p(11)$ & $p(12)$ & $p(22)$ & MAF & & & & \\
\hline & & & & Replication & 6 & 12 & 8 & 0.46 & 38 & 272 & 541 & 0.20 & 9.67E-05 & 8.13 & 2.83 & 23.30 \\
\hline & & & & Combined & 13 & 23 & 31 & 0.37 & 66 & 569 & 1074 & 0.21 & $7.46 \mathrm{E}-08$ & 6.95 & 3.43 & 14.08 \\
\hline \multirow[t]{2}{*}{18} & 48801249 & rs4078288 & G & GWAS & 7 & 11 & 23 & 0.30 & 27 & 291 & 539 & 0.20 & 5.00E-05 & 7.71 & 2.87 & 20.67 \\
\hline & & & & Combined & 12 & 24 & 31 & 0.36 & 65 & 561 & 1082 & 0.20 & 3.19E-07 & 6.66 & 3.22 & 13.77 \\
\hline
\end{tabular}

Abbreviations: A1, minor allele; CHR: chromosome; GC, gallbladder cancer; L95, lower boundary of 95\% confidence interval; MAF, minor allele frequency; OR, odds ratio; $p(11)$, number of subjects with homozygous genotypes for minor allele; $p(12)$, number of subjects with heterozygous genotypes; $p(22)$, number of subjects with homozygous genotypes for major allele; SNP, single-nucleotide polymorphism; U95, upper boundary of $95 \%$ confidence interval.

Although the pathophysiological role of DCC in gallbladder carcinogenesis has not been clarified, DCC was demonstrated to induce apoptosis in the absence of its ligand netrin-1. ${ }^{18}$ Furthermore, enforced expression of netrin 1 in mouse gastrointestinal tract has been found to induce spontaneous formation of hyperplastic and neoplastic lesions, highlighting the potential role of $D C C$ as a tumorsuppressor gene. ${ }^{19}$

Our finding that SNPs in DCC were associated with GC susceptibility supports findings of several recent studies, which also pointed out a tumor-suppressing role of the gene in gallbladder carcinogenesis. For instances, previous reports have revealed the incidences of loss of heterozygosity at DCC in GC to be as high as $30-45 \% .^{16,19,20}$ Chromosomal loss in human genome often implies that the affected region may harbor a tumor-suppressor gene, where the loss of which could lead to carcinogenesis. Nevertheless, both in silico and eQTL analysis indicated that SNPs in DCC showed no association with expression of the gene, implying other unknown mechanisms might be in action. Subsequent fine-mapping and resequencing for this region and functional analysis would be necessary to clarify association between DCC and GC.

In the current study, because of the limited number of subjects examined, we might not have enough statistical power to detect other genetic variants with modest or weak effects on susceptibility to GC. Hence, some SNPs of clinical importance might be missed in this study. Further replication study involving a larger number of samples and functional analysis of the DCC is urgently needed for validation of the association of $D C C$ with the risk of GC.

\section{CONFLICT OF INTEREST}

The authors declare no conflict of interest.

\section{ACKNOWLEDGEMENTS}

We express our heartfelt gratitude to all the subjects who participated in this study, and the members of the Rotary Club of Osaka-Midosuji District 2660 Rotary International in Japan for supporting our study. We would also like to acknowledge members of the Laboratory for Genotyping Development, RIKEN Center for Genomic Medicine for their excellent technical assistance, and the BioBank Japan for providing DNA samples and clinical information for this study. We also thank Drs Jin-Young Jang, Yoon-Sup Song, Jae-Hyun Park and Siew-Kee Low for their kind support and helpful discussion. This work was supported by Leading Project for Personalized Medicine in Ministry of Education, Culture, Sports, Science and Technology, Japan.
1 Misra, S., Chaturvedi, A., Misra, N. C. \& Sharma, I. D. Carcinoma of the gallbladder. Lancet. Oncol. 4, 167-176 (2003).

2 Piehler, J. M. \& Crichlow, R. W. Primary carcinoma of the gallbladder. Surg. Gynecol. Obstet. 147, 929-942 (1978).

3 Reid, K. M., Ramos-De la Medina, A. \& Donohue, J. H. Diagnosis and surgical management of gallbladder cancer: a review. J. Gastrointest. Surg. 11, 671-681 (2007).

4 Randi, G., Franceschi, S. \& La Vecchia, C. Gallbladder cancer worldwide: geographical distribution and risk factors. Int. J. Cancer. 118, 1591-1602 (2006).

5 Lazcano-Ponce, E., Miquel, J., Muñoz, N., Herrero, R., Ferrecio, C., Wistuba, I. et al. Epidemiology and molecular pathology of gallbladder cancer. CA Cancer J. Clin. 51, 349-364 (2001).

6 Gourgiotis, S., Kocher, H. M., Solaini, L., Yarollahi, A., Tsiambas, E. \& Salemis, N. S. Gallbladder cancer. Am. J. Surg. 196, 252-264 (2008).

7 Goldin, R. \& Roa, J. Gallbladder cancer: a morphological and molecular update. Histopathology 55, 218-229 (2009)

8 Purcell, S., Neale, B., Todd-Brown, K., Thomas, L., Ferreira, M. A., Bender, D. et al. PLINK: a tool set for whole-genome association and population-based linkage analyses. Am. J. Hum. Genet. 81, 559-575 (2007).

9 International HapMap Consortium. The International HapMap Project. Nature 426, 789-796 (2003).

10 Stranger, B. E., Forrest, M. S., Dunning, M., Ingle, C. E., Beazley, C., Thorne, N. et al. Relative impact of nucleotide and copy number variation on gene expression phenotypes. Science 315, 848-853 (2007).

11 Fearon, E. R., Cho, K. R., Nigro, J. M., Kern, S. E., Simons, J. W., Ruppert, J. M. et al. Identification of a chromosome $18 \mathrm{q}$ gene that is altered in colorectal cancers. Science 247, 49-56 (1990).

12 Vogelstein, B., Fearon, E. R., Hamilton, S. R., Kern, S. E., Preisinger, A. C., Leppert, M. et al. Genetic alterations during colorectal-tumor development. N. Engl. J. Med. 319, 525-532 (1988).

13 Uchino, S., Tsuda, H., Noguchi, M., Yokota, J., Terada, M., Saito, T. et al. Frequent loss of heterozygosity at the DCC locus in gastric cancer. Cancer Res. 52, 3099-3102 (1992).

14 Miyake, S., Nagai, K., Yoshino, K., Oto, M., Endo, M. \& Yuasa, Y. Point mutations and allelic deletion of tumor suppressor gene DCC in human esophageal squamous cell carcinomas and their relation to metastasis. Cancer Res. 54, 3007-3010 (1994).

15 Hohne, M. W., Halatsch, M. E., Kahl, G. F. \& Weinel, R. J. Frequent loss of expression of the potential tumor suppressor gene DCC in ductal pancreatic adenocarcinoma. Cancer Res. 52, 2616-2619 (1992).

16 Wistuba, I. I., Sugio, K., Hung, J., Kishimoto, Y., Virmani, A. K., Roa, I. et al. Allelespecific mutations involved in the pathogenesis of endemic gallbladder carcinoma in Chile. Cancer Res. 55, 2511-2515 (1995).

17 Yoshiya, G., Takahata, T., Hanada, N., Suzuki, K., Ishiguro, A., Saito, M. et al. Influence of cancer-related gene polymorphisms on clinicopathological features in colorectal cancer. J. Gastroenterol. Hepatol. 23, 948-953 (2008).

18 Mehlen, P., Rabizadeh, S., Snipas, S. J., Assa-Munt, N., Salvesen, G. S. \& Bredesen, D. E. The DCC gene product induces apoptosis by a mechanism requiring receptor proteolysis. Nature 395, 801-804 (1998).

19 Mazelin, L., Bernet, A., Bonod-Bidaud, C., Pays, L., Arnaud, S., Gespach, C. et al Netrin-1 controls colorectal tumorigenesis by regulating apoptosis. Nature $431,80-84$ (2004).

20 Hidaka, E., Yanagisawa, A., Sakai, Y., Seki, M., Kitagawa, T., Setoguchi, T. et al. Losses of heterozygosity on chromosomes $17 p$ and $9 p / 18 q$ may play important roles in early and advanced phases of gallbladder carcinogenesis. J. Cancer Res. Clin. Oncol. 125, 439-443 (1999).

Supplementary Information accompanies the paper on Journal of Human Genetics website (http://www.nature.com/jhg) 